\title{
Excitation patterns in the starling cochlea: A population study of primary auditory afferents
}

\author{
Otto Gleich ${ }^{\text {a) }}$ \\ Institut für Zoologie der Technischen Universität München, Lichtenbergstr. 4, 85747 Garching, Germany
}

(Received 14 April 1993; revised 10 August 1993; accepted 23 August 1993)

\begin{abstract}
The excitatory effect of different test stimuli was quantified in a large sample of primary auditory units of the starling. In order to construct an excitation pattern, the level of excitation in many single units in response to a specific test stimulus was plotted as a function of their characteristic frequency (CF). The effects of stimulus frequency and stimulus sound-pressure level on the shapes of the excitation patterns were characterized by measuring excitation patterns in response to 33 different test stimuli which covered a range of frequencies $(0.125$ to $2.0 \mathrm{kHz})$ and sound-pressure levels ( 20 to $90 \mathrm{~dB}$ SPL). In contrast to the mammalian situation, excitation patterns in the starling showed a systematic asymmetry with the high-frequency side being, on average, twice as steep as the low-frequency side. In addition, the sound-pressure level had no systematic effect either on the symmetry or on the high- and low-frequency slopes of the flanks of the excitation patterns. Thus, the nonlinear growth of excitation with increasing sound-pressure level that is typical for the high-frequency flank of mammalian excitation patterns, was not found in the starling. The differences in mammalian and avian excitation patterns are probably related to systematic differences in tuning characteristics of the respective hearing organs.
\end{abstract}

PACS numbers: 43.64.Nf, 43.64.Pg, 43.80.Lb

\section{INTRODUCTION}

The cochlea of mammals has been studied by investigating the vibration of the basilar membrane (Békésy, 1960; Johnstone and Boyle, 1967; Wilson and Johnstone, 1975; Nuttal et al., 1991), the response characteristics of hair cells (Russel and Sellick, 1983; Dallos, 1985), and the properties of auditory nerve fibers (e.g. Kiang et al., 1965). An overwhelming amount of data are available characterizing the responses of single primary auditory afferents, in much detail, with a number of different stimuli for a variety of vertebrate species (reviews: Manley, 1990, 1983). In contrast to focusing on a single cochlear location, another approach was to describe the population response of many auditory nerve fibers to a limited set of test stimuli (e.g. Pfeiffer and Kim, 1975; Kim and Molnar, 1979; Shofner and Sachs, 1986; Delgutte, 1990; Kim et al., 1990). The results of such population studies are so-called excitation patterns that are analogous to the excitation patterns described in psychophysical studies (Egan and Hake, 1950; Moore, 1988; Zwicker, 1970), and plot the distribution of neural excitation (based of some sort of predefined criterion) as a function of the characteristic frequency (CF) of the studied fiber. Because of the tonotopic cochlear organization, these plots resemble the distribution of excitatory activity along the cochlea.

Most population studies have been performed in cats for the $1 \mathrm{kHz}$ area and they demonstrate similar results for the effect of sound-pressure level on the shape of the excitation pattern, independent of the response criteria used

a) Present address: ENT-Department, University Regensburg, Postfach, 93042 Regensburg, Germany.
(Delgutte, 1990; Kim et al., 1990; Shofner and Sachs, 1986; Kim and Molnar, 1979). At low levels, the excitation was localized to a restricted area of the cochlea. An increase of the stimulus level caused an increase of overall excitation with an overproportional growth of excitation for frequencies above the test frequency. This spread of excitation (Moore, 1988) causes a substantial asymmetry of the excitation pattern at higher stimulus levels, with the slope of the flank on the high-frequency side becoming considerably shallower than that of the low-frequency side. The findings of these population studies are in accordance with results from psychophysically determined masking patterns which can also be interpreted as resembling excitation patterns (Moore, 1988).

Excitation patterns gained some attention because the effects of stimulus parameters on excitation patterns have been incorporated into models for the explanation of psychophysical phenomena (e.g. intensity discrimination; Zwicker, 1970; Florentine and Buus, 1981).

To date, the equivalent of avian excitation patterns have only been determined psychophysically by simultaneous masking in the parakeet (Saunders et al., 1978; Saunders and Pallone, 1980). However, the parakeet shows a prominent specialization with improved spectral resolving power above $1-2 \mathrm{kHz}$ as compared to other birds (Okanoya and Dooling, 1987). Thus the aim of the present study was to determine the effects of stimulus parameters on excitation patterns in the starling (a not specialized songbird; Manley and Gleich, 1992) in order to obtain comparative data. Because behavioral data on the auditory performance of the starling are available (e.g., Klump and Baur, 1990), models explaining psychophysical phenom- 
ena that are based on excitation patterns can be tested for their validity in the starling.

\section{MATERIAL AND METHODS}

The activity of auditory afferents was recorded in anaesthetized birds and has been previously described in detail (Manley et al., 1985; Gleich, 1989; Klump and Gleich, 1991). It is briefly outlined below. Birds were anaesthetized by an initial dose of nembutal $(80-90 \mathrm{mg} / \mathrm{kg}$ pentobarbital-Na), artificially respirated, and kept at a constant body temperature of $40^{\circ} \mathrm{C}$ with an automated heating system. A deep level of anaesthesia was maintained throughout the experiment by additional doses of nembutal (40-50 mg/kg) as necessary, usually every $30-60 \mathrm{~min}$. Standard techniques were applied to record the electrical activity of single cells with high impedance glass micropipettes. Data were collected using two approaches for recording from auditory units. In the first series of experiments the cochlear ganglion was accessed dorsolaterally through scala tympani ( 24 birds, 84 units; Manley et al., 1985) and in the second series of experiments primary auditory fibers were recorded in the trunk of the VIIIth nerve where it entered the brain stem after aspiration of the cerebellum ( 9 birds, 81 units; Klump and Gleich, 1991).

Acoustic stimuli were presented via a closed sound system consisting of an earphone (AKG DKK 32) and a calibrated microphone (Brüel \& Kjaer 4133) for monitoring the stimulus near the eardrum. The earpiece was fit tightly into the external earcanal of the bird and calibrated before each experiment. The output was flat within $+/-3$ $\mathrm{dB}$ in the frequency range between 0.05 and $4 \mathrm{kHz}$. At 90 $\mathrm{dB}$ SPL the second harmonic was more than $60 \mathrm{~dB}$ and the third harmonic more than $50 \mathrm{~dB}$ below the fundamental.

The response of the units was characterized by presenting a matrix of test stimuli varying in frequency and sound-pressure level that has also been called the spectral response plot (Evans, 1978; Kaltenbach and Saunders, 1987; Klump and Gleich, 1991). Test frequencies typically covered a range of 2-3 oct around the CF (frequency of the highest sensitivity) in $0.1-0.2$-oct steps. The sound pressure was tested from well below to well above unit threshold, generally from 10 to $90 \mathrm{~dB}$ SPL in 4 or 5 -dB steps. The test stimuli consisted of 100 -ms tone pips with 2.5-ms rise and fall times. The repetition rate was 4 per second and each test stimulus was presented twice. Although the high repetition rate can affect the response at high levels in some cells (Manley et al., 1985; Relkin and Doucet, 1991), due to incomplete recovery, it was used as a compromise between the restricted recording time and an optimal characterization of the cells. Furthermore incomplete recovery would mainly affect the responses of cells with CFs close to the test frequency and at high stimulus levels. However, the independence of the excitation pattern shape from the stimulus level (see Sec. II) argues that the relatively high repetition rate did not significantly affect the measurements. The number of action potentials that occurred during each stimulus presentation was registered. Thus a 200-ms response sample was collected for each point of the frequency-sound-pressure matrix. A typical
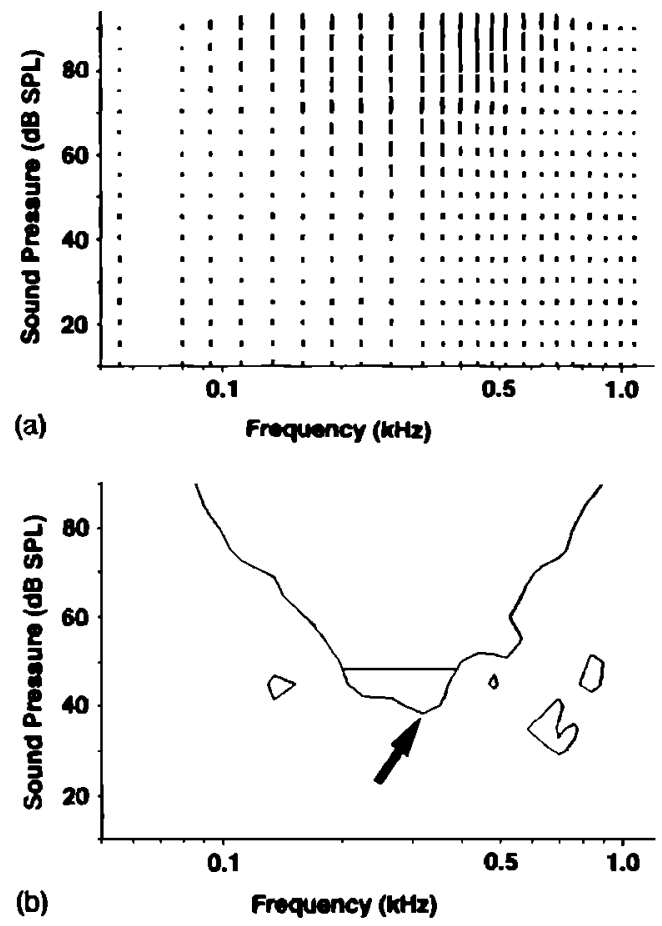

FIG. 1. Response characteristic of an auditory afferent to a matrix of test stimuli. (a) The rate measured during stimulus presentation is indicated by the height of the bars. The bottom of each bar represents frequency and sound pressure of the stimulus. The area with the high bars indicates the excitatory response area of the cell. (b) Isorate contour calculated from the data shown above with a criterion just above the spontaneous discharge rate. This represents the tuning curve of the cell, with CF 0.32 $\mathrm{kHz}$ ) and threshold (38.4 dB SPL) indicated by an arrow at the tip of the curve. The bandwidth used to calculate the $Q_{10} \mathrm{~dB}$ value (1.69) is also shown.

representation of such a matrix is illustrated in Fig. $1(a)$ as a bar graph with the height of the bars representing the number of spikes registered during the two 100-ms tone presentations. The area with the high bars is the excitatory response area of the cell. An isorate contour was calculated from such data sets as a threshold tuning curve [Fig. 1(b); Gleich and Narins, 1988] in order to determine the CF, threshold at CF [arrow in Fig. 1(b)], and the $Q_{10} \mathrm{~dB}$ value (CF/bandwidth $10 \mathrm{~dB}$ above threshold).

The aim of this study was to determine the effects of test frequency and sound pressure on the excitation pattern in the starling cochlea. Thus the responses of a large sample of neurons were measured for as many stimuli as possible from a matrix of $\mathbf{4 5}$ test stimuli ranging in frequency from 0.125 to $2 \mathrm{kHz}$ (in octave steps) and in sound pressure from 10 to $90 \mathrm{~dB}$ SPL (in 10-dB steps). From the spectral response plot of each cell [e.g., Fig. 1(a)] rateintensity functions were determined for the $\mathrm{CF}$ and the test frequencies $0.125,0.25,0.5,1.0$, and $2.0 \mathrm{kHz}$ [if they were available; Fig. 2(a)]. Then, in order to obtain a measure for the excitation elicited by a test stimulus, the sound pressure of a CF tone was determined that elicited the same discharge rate as the test stimulus under investigation [Fig. 2(b); method in Moore, 1988]. The sound pressure of the $\mathrm{CF}$ tone that elicited the same discharge rate as the test tone was termed the CF-level equivalent. The CF-level 

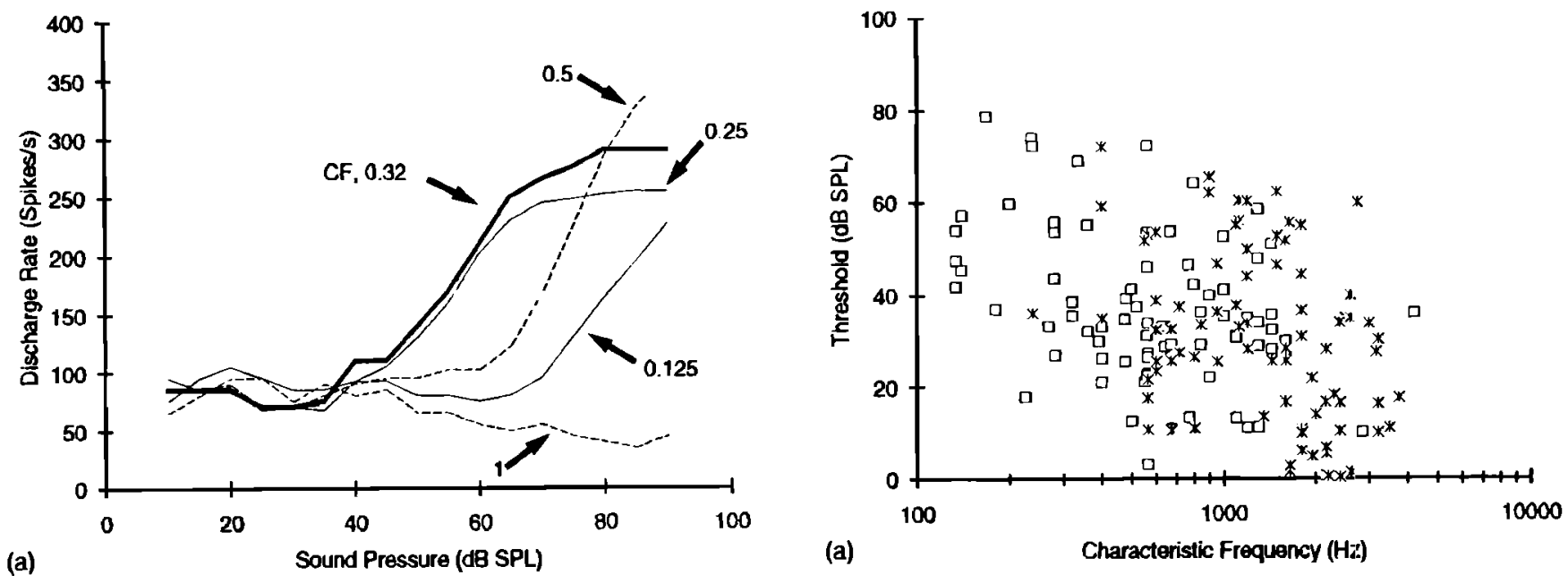

(a)

Characteristic Frequency $\left(\mathrm{H}^{\prime \prime}\right)$
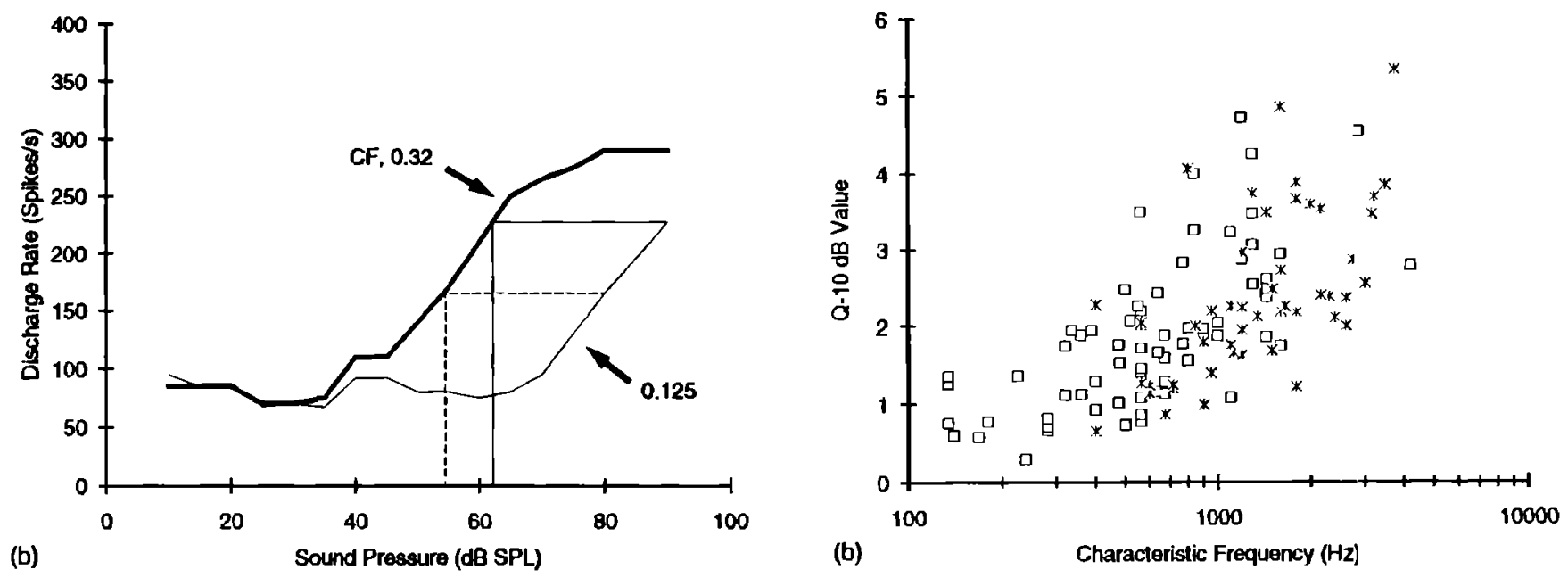

FIG. 2. Input-output curves obtained at different frequencies. The frequencies of individual rate-intensity functions are indicated by the numbers in the figure. The rate-intensity function obtained at the CF is emphasized by the heavy line. Thin continuous lines code for test frequencies below and dashed lines for test frequencies above the CF. (a) The 0.5$\mathrm{kHz}$ test stimulus at the highest sound pressures evoked a higher discharge rate than the $\mathrm{CF}$ stimulus. The $\mathrm{I}-\mathrm{kHz}$ stimulus induced primary suppression, the rate was clearly reduced below spontaneous rate for sound pressures above $60 \mathrm{~dB}$ SPL. (b) The determination of CF-level equivalents is illustrated. The sound pressure of a CF stimulus that elicits the same rate as a test stimulus is determined. The discharge rate elicited by a $90-\mathrm{dB}, 0.125-\mathrm{kHz}$ test stimulus is the same as that of a $62.2-\mathrm{dB}$ stimulus at $\mathrm{CF}$ (indicated by the continuous line). Thus the CF-level equivalent for this test stimulus was $62.2 \mathrm{~dB}$ SPL. The CF-level equivalent of a $80-\mathrm{dB}, 0.125-\mathrm{kHz}$ test stimulus was $54.5 \mathrm{~dB}$ (indicated by the dashed line).

equivalent was determined only for suprathreshold test stimuli. With this type of analysis, each cell provided data only for the test stimuli within it's excitatory response area, which means that only cells that were excited contributed to the excitation pattern.

Two complications were encountered with this type of analysis. The first was found primarily in low-CF cells. Here in some cases, test stimuli above CF caused higher discharge rates than the maximal rates elicited at the $\mathbf{C F}$ [0.5 kHz in Fig. 2(a)]. No CF-level equivalent could be measured in these cases. The second complication was introduced by the phenomenon of primary suppression 


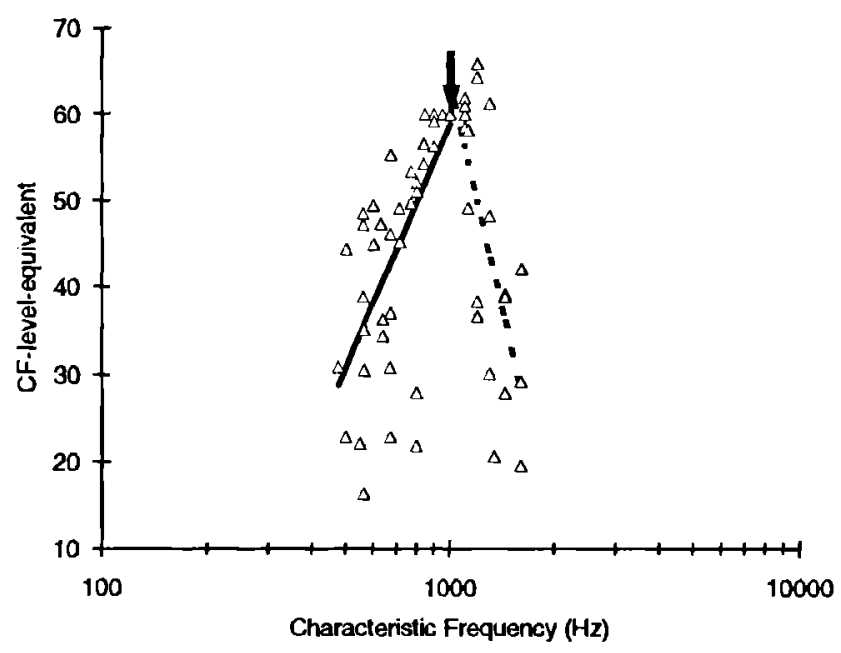

FIG. 4. Excitation pattern constructed from the responses of 63 auditory afferents to a $1-\mathrm{kHz} 60-\mathrm{dB}$ SPL stimulus. Maximum excitation was around the test stimulus (indicated by the arrow), with excitation decreasing toward lower and higher frequencies. Regressions are shown which were calculated through cells with CFs below $1 \mathrm{kHz}$ (continuous line; $n=40 ; r=0.67 ; p<0.001$ ) and above $1 \mathrm{kHz}$ (dashed line; $n=23$; $r=-0.74 ; p<0.001$ ). The slope of the regression through the lowfrequency flank of this excitation pattern is $28.1 \mathrm{~dB} /$ oct and is -51.6 $\mathrm{dB} /$ oct for the high-frequency flank.

$0.5-$ and $1-\mathrm{kHz}$ stimuli, with the highest density of cells around $1 \mathrm{kHz}$.

The distribution of CF and threshold [Fig. 3(a)] and $\mathrm{CF}$ and $Q_{10} \mathrm{~dB}$ value [Fig. 3(b)] were very similar to previously published starling data (Manley et al., 1985; Klump and Gleich, 1991). The different approaches for recording from primary afferents are coded by different symbols. Open squares represent the ganglion recordings, while crosses represent cells recorded from the trunk of the eighth nerve. The two approaches differed with respect to the CF range sampled. Low-CF cells were more easily accessed in the cochlear ganglion, while high-CF cells were preferentially sampled in the nerve trunk. In the CF range around $1 \mathrm{kHz}$, the data collected at the two different recording sites overlapped completely. Similar results were obtained with respect to other tuning characteristics such as high- and low-frequency slopes of the tuning curves. Because the data from the two recording sites differed only with respect to the CF range, they were pooled and not differentiated for further analysis.

Excitation patterns were constructed by plotting the CF-level equivalent versus the CF (Fig. 4) which represents the position along the papilla basilaris (Gleich, 1989). The example in Fig. 4 obtained with a $1-\mathrm{kHz}$ stimulus of $60 \mathrm{~dB}$ SPL demonstrates the typical features. Each triangle shows the excitatory activity of one single cell in response to the test stimulus, which is indicated by the arrow. Excitation was highest for cells with CFs around 1 $\mathrm{kHz}$ and declined toward lower and higher CFs, respectively, indicating that the test stimulus excited only a restricted area of the basilar papilla. In order to characterize the shape of the excitation pattern more systematically, regression lines were calculated through data from cells with a CF below and above the test frequency respectively, as illustrated in Fig. 4. If the correlation of CF and CFlevel equivalent was significant, the slopes of these regression lines were accepted as a measure of the steepness of the low- and high-frequency flanks of the excitation pattern. The correlation was generally significant if enough data that spanned a sufficient CF range were available. This was the case for excitation patterns at higher stimulus levels that were 10 or $20 \mathrm{~dB}$ above the threshold of the most sensitive cells near the test frequency. With this method, the steepness of the low- and high-frequency side of 17 excitation patterns were determined. For six excitation patterns only the low- and in another six only the high-frequency side could be characterized. Neither the low-nor the high-frequency flank could be analyzed in four excitation patterns due to an insufficient number of data points.

The effects of test frequency and test sound pressure are qualitatively illustrated in Fig. 5, which shows nine examples of the 33 excitation patterns that have been constructed. Each point on the graphs represents the excitatory activity of one cell in response to the test stimulus, which is indicated on top of each graph. The maximum excitation followed the test frequency. Low test frequencies $(0.5 \mathrm{kHz}$, the bottom row in Fig. 5) excited most effectively low-CF neurons and thus apical positions in the basilar papilla, while high test frequencies $(2 \mathrm{kHz}$, the top row in Fig. 5) were most effective in higher-CF cells which innervate more basal areas of the basilar papilla (Gleich, 1989). These graphs represent the distribution of excitatory activity along the basilar papilla in response to a test stimulus. The width of the excitation pattern apparently increased from high (top row Fig. 5) to low (bottom row of Fig. 5) test frequencies on the logarithmic scale used. On a linear frequency scale, however, the bandwidth of the excitation pattern, expressed in hertz, increased with frequency (see Table I). An increase of the sound-pressure level (from the right to the left column in Fig. 5) caused an increase of the excitation with some spread of excitation toward CFs above and below the test frequency. However, in contrast to the situation in mammals (Moore, 1988), the starling data did not show a pronounced spread of excitation toward higher frequencies at high-soundpressure levels.

The qualitative findings illustrated in Fig. 5 were subjected to a three-factor analysis of variance with the steepness of the low- and high-frequency flanks of the excitation patterns as dependent variable and the low- or highfrequency side, the test frequency and the test level as independent variables. This analysis revealed that the slopes of the low- and high-frequency sides of the excitation patterns differed significantly ( $D F=1 ; F=5.9 ; p<0.05$ ). The test frequency clearly affected the slopes of the excitation patterns ( $D F=4 ; F=16.6 ; p<0.05$ ), while the level of the test stimulus had no systematic effect on the slopes ( $D F$ $=7 ; F=1.2 ; p>0.05$ ). Interactions between the independent factors could not be analyzed due to missing values. Figure 6 shows the slope of the high-frequency flank of the excitation pattern (lower part; $n=23, r=-0.81$, $p<0.001$ ) as well as the slope of the low-frequency flank 

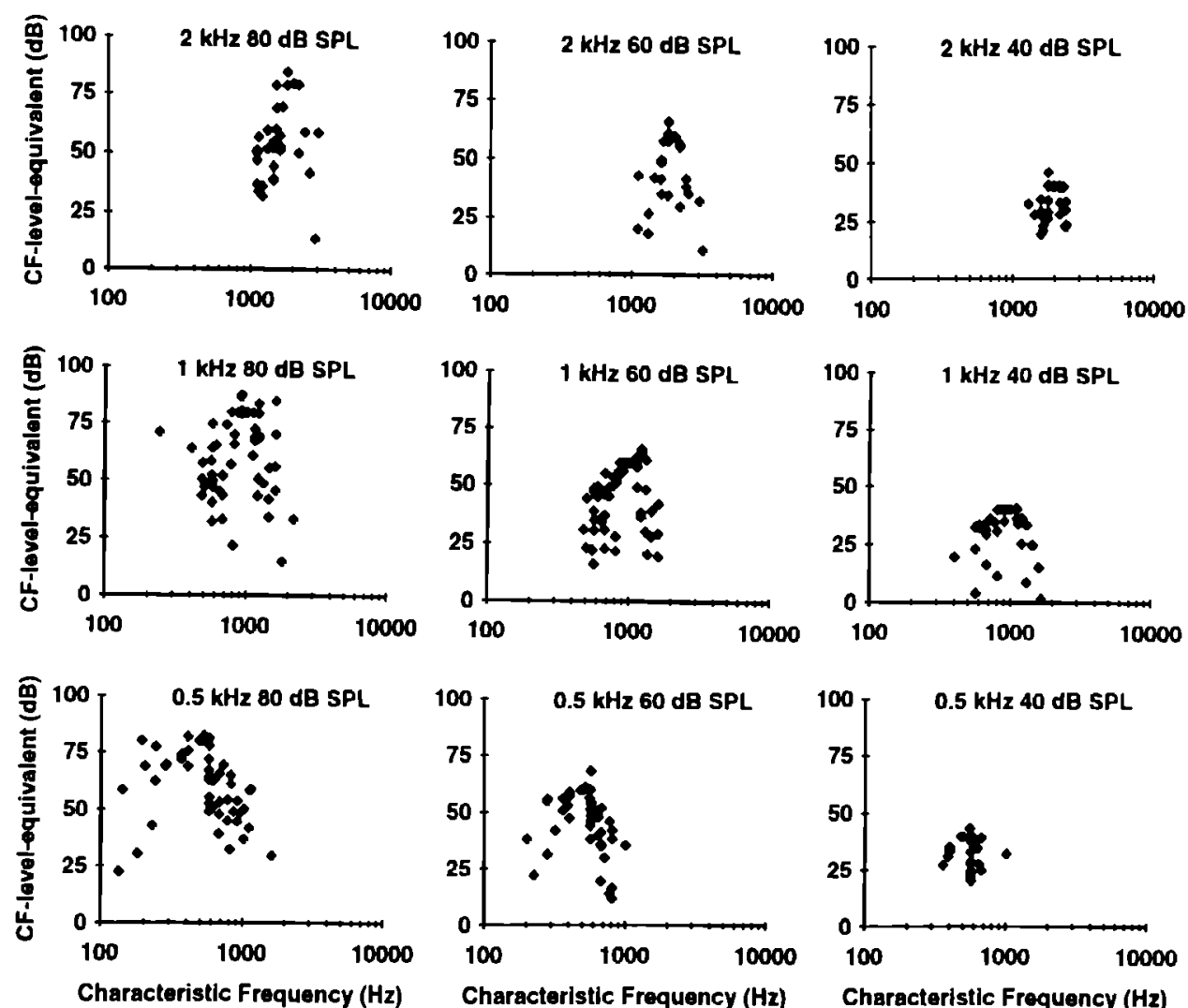

FIG. 5. Examples of excitation patterns in response to nine different test stimuli to exemplify the effect of stimulus parameters. The test frequency decreased from $2 \mathrm{kHz}$ in the top row to $0.5 \mathrm{kHz}$ in the bottom row, while the sound-pressure level decreased from $80 \mathrm{~dB} S P L$ in the left column to 40 dB SPL in the right column.

(upper part; $n=23, r=0.87, p<0.001$ ) as a function of the test frequency. The steepness of the low and high frequency flanks of the excitation pattern both increased systematically with increasing test frequency and they were significantly correlated $(n=17, r=0.52, p<0.05)$. This is in agreement with the observation that excitation patterns in response to low-frequency stimuli appeared broader than those in response to high-frequency stimuli (Fig. 5). Figure 6 also clearly demonstrates that the high-frequency flanks of the excitation patterns were systematically steeper than the low-frequency flanks. Indeed, in the 17 excitation patterns where the slopes of the low- and high-frequency flank were available, the high-frequency flanks were, on average, twice as steep as the low-frequency flanks (expressed in $\mathrm{dB} / \mathrm{oct}$ ) and the excitation patterns were thus systematically asymmetric (e.g., Fig. 4).

The effect of sound-pressure level on the shape of the excitation pattern was determined by plotting the test sound-pressure level against the low (upper part of Fig. 7; $n=23, r=-0.23, p>0.05$ ) and the high-frequency slope of the excitation pattern (lower part of Fig. 7, $n=23$, $r=0.25, p>0.05$ ). There was no systematic change of the slopes with sound pressure. This was true for the data pooled across frequencies (statistics given above) as well as for individual test frequencies. In addition, for the $17 \mathrm{ex}$ citation patterns where the slope of the high- and lowfrequency flank of the excitation pattern were available, there was no systematic influence of the sound-pressure level on the ratio of the high- and low-frequency slopes which was taken as an indicator of the symmetry of the excitation pattern $(n=17, r=-0.24, p>0.05)$. The quantitative analysis confirmed the qualitative impression gained from Fig. 5 that test sound pressure neither influenced the slopes of the high- or low-frequency flanks of the

TABLE I. Excitation by test stimuli of $0.5,1.0$, and $2.0 \mathrm{kHz}$ (labeled in the top line) at 30, 60, and $90 \mathrm{~dB}$ SPL (labeled in the left column). The lowerand upper-frequency limit $(\mathrm{kHz})$, the frequency range in octaves (oct) and the percentage of the papilla length (\%) excited by the different test stimuli are listed.

\begin{tabular}{|c|c|c|c|c|c|c|c|c|c|}
\hline & \multicolumn{3}{|c|}{$0.5 \mathrm{kHz}$} & \multicolumn{3}{|c|}{$1.0 \mathrm{kHz}$} & \multicolumn{3}{|c|}{$2.0 \mathrm{kJz}$} \\
\hline & $(\mathrm{kHz})$ & (oct) & $(\%)$ & $(\mathrm{kHz})$ & (oct) & $(\%)$ & $(\mathrm{kHz})$ & (oct) & (\%) \\
\hline $30 \mathrm{~dB}$ & $0.16-1.0$ & 2.6 & 30 & $0.48-1.7$ & 1.8 & 30 & $1.2-3.0$ & 1.3 & 32 \\
\hline $60 \mathrm{~dB}$ & $0.05-2.0$ & 5.3 & 53 & 0.23 & 3.6 & 55 & $0.7-4.6$ & 2.7 & 57 \\
\hline $90 \mathrm{~dB}$ & $0.02-4.0$ & 7.6 & 81 & $0.1-4.6$ & 5.5 & 80 & $0.4-6.6$ & 4.0 & 82 \\
\hline
\end{tabular}




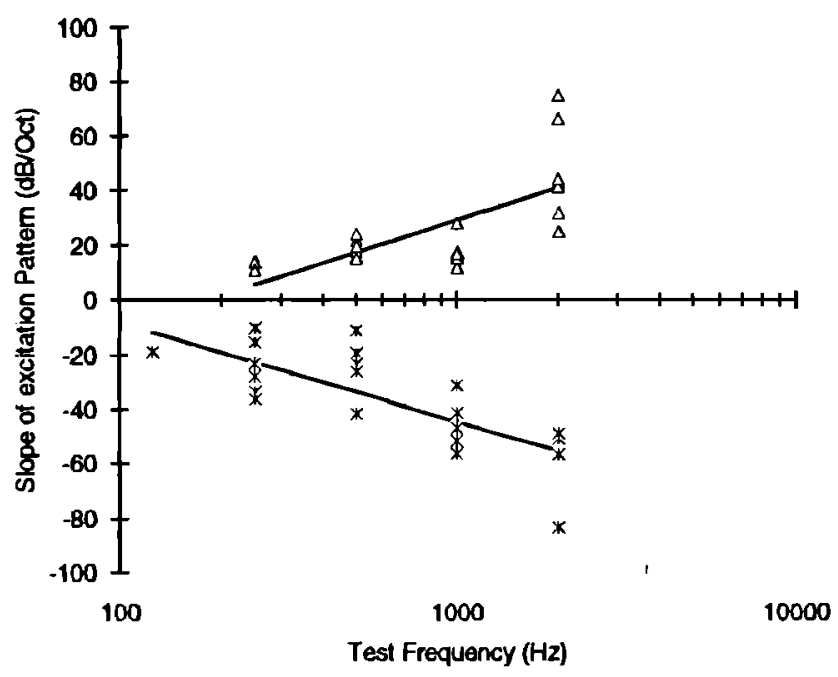

FIG. 6. The slopes of the regressions through the high- (crosses) and low- (open triangles) frequency flanks of the excitation patterns as a function of stimulus frequency. There was a highly significant tendency for the steepness of the high $(n=23 ; r=0.81 ; p<0.001)$ and low $(n=23$; $r=-0.87 ; p<0.001$ ) frequency slopes to increase with stimulus frequency. On average, the slope of the low-frequency side of the excitation pattern was only half as steep as that of the high-frequency side.

excitation patterns nor their symmetry. The independence of the excitation pattern slopes from sound-pressure level is consistent with a linear growth of excitation with increasing sound pressure (Fig. 5).

\section{DISCUSSION}

The data presented here were obtained by recording from two different locations, the cochlear ganglion and the trunk of the VIIIth nerve. Because there were no differences in the response characteristics of the two recording sites except for the accessible CF range (e.g. Fig. 3; Klump and Gleich, 1991), the results were pooled to increase the data base.

A number of different criteria have been used to obtain measures of stimulus-related activity of the responses of auditory nerve fibers in published population studies. Kim and Molnar (1979) evaluated six different response measures based on average rate and phase-locking characteristics. Kim et al. (1990) and Kim and Parham (1991) recently introduced $d^{\prime}$ (driven rate/standard deviation) to obtain excitation patterns. An alternative measure that determines the effective amount of excitation by a stimulus is outlined by Moore (1988). He suggested using the soundpressure level of a $\mathrm{CF}$ tone that produces the same neural activity as the test tone, as a value of stimulus-related excitation which is called the CF-level equivalent throughout this study. This measure is less affected by the large variability of spontaneous and maximum driven rate among auditory afferents as those based on rate criteria and was therefore adopted for the analysis of the starling excitation patterns. In addition Delgutte (1990) used a very similar procedure for the nonsimultaneously determined excitation patterns in the cat; thus cat and starling data can be easily compared.
The excitation patterns determined in this study (Figs. $4,5)$ show considerable variability in the CF-level equivalent among cells with the same CF. There are several potential sources for this variability. In order to obtain reasonable numbers of neurons to construct the excitation patterns, data from 33 birds had to be pooled. In addition the CF determination was performed with a resolution of $0.1-0.2$ oct introducing a potential error of 0.1 oct. Major factors contributing to the variability in the excitation pattern plots are, however, besides these measurement errors, the large variability of thresholds at a given $\mathrm{CF}$ [ $>60 \mathrm{~dB}$, Fig. 3(a); Manley et al., 1985] and the large variability of the slopes of rate-intensity functions of individual neurons at the different test stimuli. A similar variability of population responses, probably due to the variability of rateintensity function slopes and the saturating nonlinearity of rate-intensity functions was also present in the mammalian data (Kim and Molnar, 1979; Evans, 1981; Delgutte, 1990). However, comparing the starling's excitation patterns described in this study with the mammalian population studies, mentioned above, demonstrates differences which are clearly evident despite the scatter in avian and mammalian data sets.

The stimulus frequency was shown to have a systematic effect on the excitation pattern. The average slopes of the high- and low-frequency flanks of the excitation patterns were determined for the different test frequencies from the regression lines shown in Fig. 6. Using these slopes it was possible to calculate the average $Q_{10} \mathrm{~dB}$ value at four test frequencies. It increased with the test frequency from $0.93(0.25 \mathrm{kHz}), 1.79(0.5 \mathrm{kHz}), 2.63(1.0 \mathrm{kHz})$ to $3.45(2.0 \mathrm{kHz})$. These values are virtually identical to psychophysical data obtained in the parakeet by simultaneous masking (Saunders and Pallone, 1980).

In addition the frequency range where a specific test stimulus caused excitation (the frequency range between the intersects of the slopes of the excitation pattern with the $0-\mathrm{dB}$ excitation line) could be estimated from the data in Fig. 6. The activated ranges (in $\mathrm{kHz}$ and in octaves) for different test stimuli are given in Table I. From the frequency map of the starling basilar papilla (derived from functionally characterized, cobalt-labeled auditory afferents; Gleich, 1989) and the frequency range activated by a stimulus it was possible to estimate the distance along the papilla that was excited by a particular stimulus. The values are also given in Table $I$ as a percentage of the total papillar length. For all three test frequencies, about $30 \%$ or $0.9 \mathrm{~mm}$ were activated by the $30 \mathrm{~dB}$ SPL stimulus while the activated area increased to $55 \%(1.6 \mathrm{~mm})$ at $60 \mathrm{~dB}$ SPL and $80 \%(2.2 \mathrm{~mm})$ at $90 \mathrm{~dB}$ SPL. The physical proportion of the basilar papilla along its length which was activated by a stimulus depended on the level but appeared fairly independent of the test frequency.

The observation that twice as many octaves were activated by a $0.5-\mathrm{kHz}$ stimulus than by one of $2 \mathrm{kHz}$ corresponds quite well with the different frequency mapping constants on the basilar papilla described for these frequency ranges in the starling (Gleich, 1989). The widening of excitation patterns from high- to low-frequency stimuli 


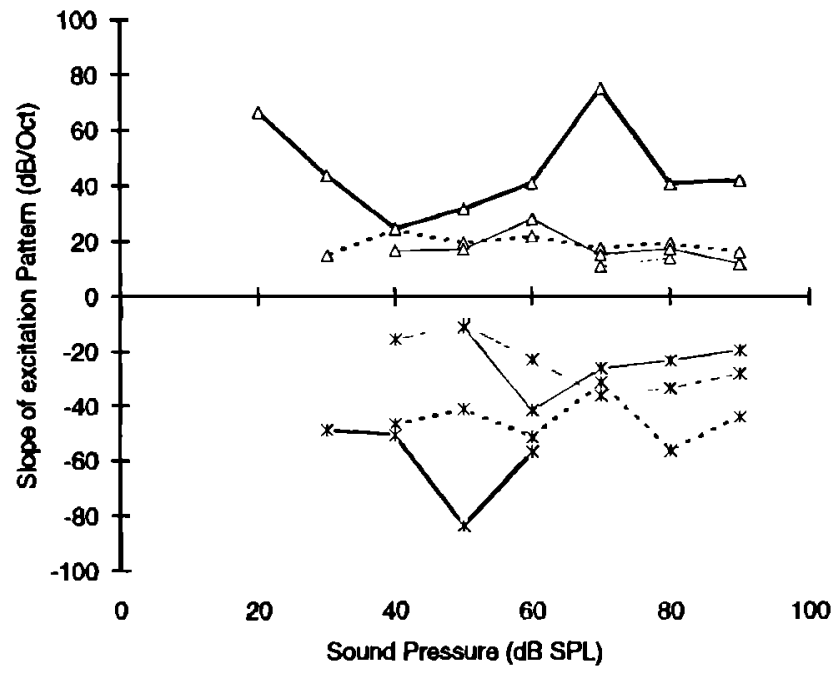

FIG. 7. The slopes of the regressions through the high- (crosses) and low- (open triangles) frequency flanks of the excitation patterns as a function of stimulus level. Different test frequencies are coded by the line pattern (heavy-continuous $=2 \mathrm{kHz}$; dashed $=1 \mathrm{kHz}$; thin-continuous $=0.5 \mathrm{kHz}$; thin-dotted $=0.25 \mathrm{kHz}$ ). The variation of sound-pressure level did not exert any systematic effect on the slopes and thus the symmetry of excitation patterns in the starling (LF: $n=23 ; r=-0.23 ; p>0.05$; HF: $n=23 ; r=0.25 ; p>0.05$ ).

on the logarithmic scale as related to stimulus frequency (Figs. 5 and 6 ) reflects the fact that the frequency distribution along the starling papilla is not logarithmic and the mapping of high-frequency octaves occupies more space on the basilar papilla than that of low-frequency octaves. In addition the sharpness of tuning increased on average with
CF (Manley et al., 1985) so that excitation patterns obtained with high test frequencies were narrower than those obtained at low test frequencies (on a logarithmic scale).

Although overall excitation increased with stimulus level (Fig. 5), the slopes of the high- and low-frequency flanks of starling excitation patterns did not systematically change with the sound-pressure level (Figs. 5,7). This is in marked contrast to the situation described for mammals. Independent of the measure of excitation used, an increase in the stimulus level caused a larger increase in excitation above the test frequency as compared to below the test frequency. This was found for excitation patterns determined using rate and phase-locking criteria ( $\mathrm{Kim}$ and Molnar, 1979; Kim et al., 1990; Shofner and Sachs, 1986), response profiles constructed from $d^{\prime}$ measures (Kim et al., 1990; Kim and Parham, 1991) and also when a measure similar to the CF-level equivalent was used (Delgutte, 1990). In addition, psychophysically determined masking patterns that are believed to resemble excitation patterns showed that an increase of the stimulus level caused a spread of excitation toward high frequencies (Moore, 1988; Egan and Hake, 1950; Zwicker, 1970) so that excitation patterns became increasingly asymmetrical with higher stimulus levels in mammals. The only comparative avian masking data available to date are those by Saunders et al. (1978) and Saunders and Pallone (1980) who determined the effects of varying sound pressure level on the shape of psychophysical masking patterns in the parakeet. Using a simultaneous masking paradigm they showed that changing the sound pressure of the masker had no effects on the slopes of the low- and high-frequency
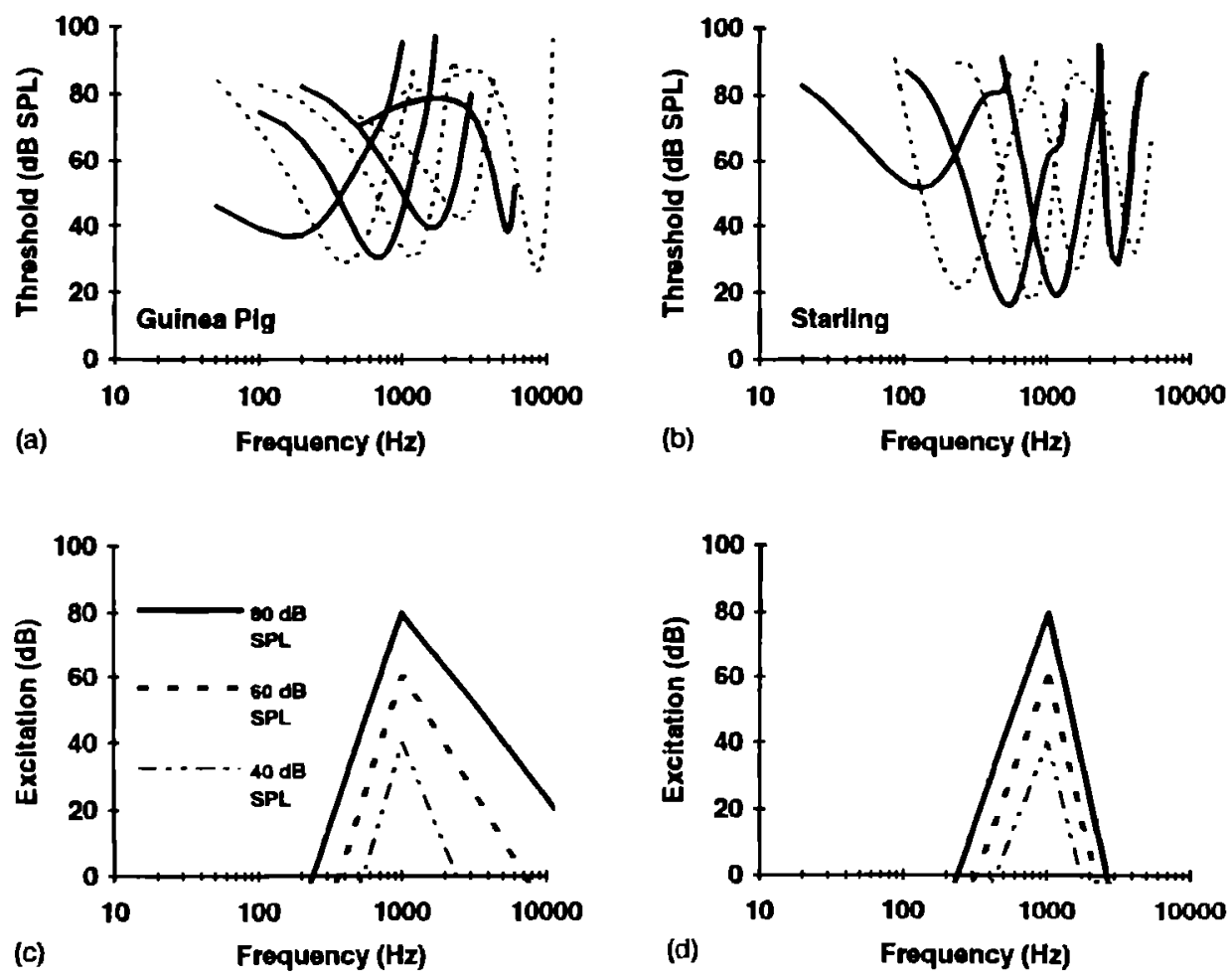

FIG. 8. Selected tuning curves from the guinea pig (a) and the starling (b) are shown to illustrate the effects of tuning-curve shape on excitation patterns that are schematically illustrated for three different levels (80,60, and $40 \mathrm{~dB}$ SPL) of a $1-\mathrm{kHz}$ stimulus for a mammal (c) and the starling (d). 
flanks of the masking patterns. Thus the level dependence of the excitation patterns in mammals, and the level independence in birds shows a fundamental difference between these groups which probably reflects differences in cochlear structures and tuning mechanisms.

An important difference between the response characteristics of avian and mammalian auditory afferents which probably influences the shape of excitation patterns is the shape of the frequency threshold curves (Manley et al, 1985). In mammals, the high-frequency flank of the neural tuning curves was systematically steeper than the lowfrequency flank, and many cells with CFs above 3-5 kHz display, below the $\mathrm{CF}$, the so-called tail. In birds, neural tuning curves were, on average, symmetrical and did not show tails. This difference is illustrated qualitatively with selected examples of the guinea pig [Fig. 8(a); my unpublished data] and the starling [Fig. 8(b)] tuning curves that cover about the same CF range. Due to the asymmetry, guinea pig fibers responded over a wider frequency range at high stimulus levels than did those of starlings. What are the consequences for excitation patterns? A low-level stimulus of $1 \mathrm{kHz}$ will excite a few cells with a characteristic frequency around $1 \mathrm{kHz}$, resulting in a localized excitation [dot-dashed lines in the sketches of Fig. 8(c) and (d)]. In the mammalian case, an increase of the stimulus level will activate more cells with $\mathrm{CFs}$ above $1 \mathrm{kHz}$ than below 1 $\mathrm{kHz}$, due to the asymmetrical shape of the neural tuning curves. The excitation pattern will become progressively asymmetrical [dotted and continuous lines in Fig. 8(c)]. In birds, due to the narrow and, on average, symmetrical neural tuning curves, the slopes of the high- and low-frequency flanks of the excitation pattern (and its symmetry) are not changed by the increase in stimulus level and excitation remains restricted to a narrower frequency range [Fig. 8(d)]. Although this simplified model does not consider the stimulus-dependent variability of the slopes and the saturating nonlinearities of the rate-intensity functions it illustrates that the different shapes of mammalian and avian tuning curves are reflected in the properties of the excitation patterns. The observation, that the highfrequency flanks of the excitation patterns in the starling were on average twice as steep as the low-frequency flanks is in contrast to the finding that single-cell tuning curves are, on average, symmetrical. However, the tuning curve symmetry varied systematically with $\mathrm{CF}$, cells with a $\mathrm{CF}$ below $1 \mathrm{kHz}$ tended to have steeper low- than highfrequency flanks (Manley et al., 1985). In addition the slopes of the tuning curve flanks tended to increase with CF. These two factors probably contribute to the systematic, level-independent asymmetry of excitation patterns in the starling.

The differences between mammalian and avian excitation patterns also have consequences for models that use characteristics of excitation patterns to explain psychophysically observed phenomena. This will only briefly be discussed for some aspects of intensity discrimination. Zwicker (1970) proposed a model, based on the nonlinear spread of excitation toward high frequencies, to explain the improved intensity discrimination at high as compared to low stimulus levels. Klump and Baur (1990) determined intensity discrimination ability in the starling and compared it with data from other birds and from mammals. They found that, as in mammals, the ability of starlings to discriminate intensity differences improves with an increase of overall level. In the starling excitation patterns did not show nonlinear growth and increased asymmetry with increased stimulus levels (Figs. 5, 7) for stimulus parameters where these nonlinearities were very prominent in the mammal (Kim and Molnar, 1979; Kim et al., 1990; Kim and Parham, 1991; Delgutte, 1990; Shofner and Sachs, 1986). Thus Zwickers simple excitation pattern model does not explain the improved intensity discrimination observed at high levels in the starling (Klump and Baur, 1990). It remains to be investigated if modified models based on excitation patterns (Florentine and Buus, 1981) can account for the performance of starling intensity discrimination. Alternatively, models based on the optimal combination of the information of relatively small numbers of auditory afferents (Sachs and Abbas, 1974; Winter and Palmer, 1991) which have been formulated for mammals might, after appropriate modification, be applied to explain intensity discrimination in birds.

\section{ACKNOWLEDGMENTS}

This work was supported by a Helmholtz grant and the SFB 204 (Gehör). I thank G. A. Manley and G. M. Klump for encouragement and discussion during the project and comments on the manuscript. I thank M. Florentine and an anonymous reviewer for their comments on the manuscript. G. Hinderer instructed me in the use of the "SPSS for Windows" statistical software package. I also thank G. A. Manley and the staff of the zoology department of the Technical University Munich for providing a productive and enjoyable working environment.

von Bèkèsy, G. (1960). Experiments in Hearing (McGraw-Hill, New York).

Dallos, P. (1985). "Response characteristics of mammalian outer hair cells," J. Neurosci. 5, 1591-1608.

Delgutte (1990). "Physiological mechanisms of psychophysical masking: Observations from auditory-nerve fibers," J. Acoust. Soc. Am. 87, 791809.

Dooling, R. J., Okanoya, K., Downing, J., and Hulse, S. (1986). "Hearing in the starling (Sturnus vulgaris): Absolute thresholds and critical ratios," Bull. Psychonom. Soc. 24, 462-466

Egan, J. P., and Hake, H. W. (1950). "On the masking pattern of a simple auditory stimulus," J. Acoust. Soc. Am. 22, 622-630.

Evans, E. F. (1978). "Place and time coding of frequency in the peripheral auditory system: so.ne physiological pros and cons," Audiology 17, $369-420$.

Evans, E. F. (1981). "The dynamic range problem: Place and time coding at the level of cochlear nerve and nucleus," in Neuronal Mechanisms of Hearing, edited by J. Syka and L. Aitkin (Plenum, New York), pp. 69-85.

Florentine, M., and Buus, S. (1981). "An excitation-pattern model for intensity discrimination," J. Acoust. Soc. Am. 70, 1646-1654.

Gleich, O. (1989). "Auditory primary afferents in the starling: Correlation of function and morphology," Hear. Res. 37, 255-268.

Gleich, O., and Narins, P. M. (1988). "The phase response of primary auditory afferents in a songbird (Stumus vulgaris $L$ )," Hear. Res. 32, 81-92.

Johnstone, B. M., and Boyle, A. J. F. (1967). "Basilar membrane vibration examined with the Mössbauer technique," Science 158, 389-390. 
Kaltenbach, J. A., and Saunders, J. C. (1987). "Spectral and temporal response patterns of single units in the chinchilla dorsal cochlear nucleus," Exp. Neurol. 96, 406-419.

Kiang, N. Y. S., Watanabe, T., Thomas, E. C., and Clark, L. F. (1965). Discharge patterns of single fibres in the cat's auditory neve, Res. Monogr. No. 35 (MIT Press, Cambridge, MA).

Kim, D. O., and Molnar, C. E. (1979). “A population study of cochlear nerve fibers: comparison of spatial distributions of average-rate and phase-locking measures of response to single tones," J. Neurophys. 42, 16-30.

Kim, D. O., and Parham, K. (1991). "Auditory nerve spatial encoding of high-frequency pure tones: population response profiles derived from $D^{\prime}$ measure associated with nearby places along the cochlea," Hear. Res. 52, 167-180.

Kim, D. O., Chang, S. O., and Sirianni, J. G. (1990). "A population study of auditory-nerve fibers in unanesthetized decerebrate cats: $\mathbf{R e}$ sponse to pure tones," J. Acoust. Soc. Am. 87, 1648-1655.

Klump, G. M., and Baur, A. (1990). "Intensity discrimination in the european starling (Sturnus vulgaris)," Naturwissenschaften 77, 545 548.

Klump, G. M., and Gleich, O. (1991). "Gap detection in the european starling (Sturnus vulgaris). III. Processing in the peripheral auditory system," J. Comp. Physiol. A 168, 469-476.

Manley, G. A. (1983). "Auditory nerve fibre activity in mammals," in Bioacoustics, edited by B. Lewis (Academic, London,), pp. 207-232.

Manley, G. A. (1990). Peripheral Hearing Mechanisms in Reptiles and Birds (Springer-Verlag, Berlin)

Manley and Gleich (1992). "Evolution and specialization of function in the avian auditory periphery," in The Evolutionary Biology of Hearing, edited by D. B. Webster, R. R. Fay, and A. N. Popper (SpringerVerlag, New York), pp. 561-580.

Manley, G. A., Gleich, O., Leppelsack, H.-J., and Oeckinghaus, H. (1985). "Activity patterns of cochlear ganglion neurones in the starling," J. Comp. Physiol. A 157, 161-181.

Moore, B. C. J. (1988). An Introduction to the Psychology of Hearing (Academic, New York).
Nuttall, A. L., Dolan, D. F., and Avinash, G. (1991). “Laser Doppler velocimetry of basilar membrane vibration," Hear. Res. 51, 203-214.

Okanoya, K., and Dooling, R. J. (1987). "Hearing in passerine and psittacine birds: A comparative study of absolute and masked auditory thresholds," J. Comp. Psychol. 101, 7-15.

Pfeiffer, R. R., and Kim, D. O. (1975). "Cochlear nerve fibre responses: Distribution along the cochlear partition," J. Acoust. Soc. Am. 58, 867-869.

Relkin, E. M., and Doucet, J. R. (1991). "Recovery from prior stimulation. I: Relationship to spontaneous firing rates of primary auditory neurons," Hear. Res. 55, 215-222.

Russell, I. J., and Sellick, P. M. (1983). "Low-frequency characteristics of intracellularly recorded receptor potentials in guinea-pig cochlear hair cells," J. Physiol. 338, 179-206.

Sachs, M. B., and Abbas, P. J. (1974). "Rate versus level functions for auditory-nerve fibers in cats: Tone-burst stimuli," J. Acoust. Soc. Am. 56, $1835-1847$.

Saunders, J. C., and Pallone, R. L. (1980). "Frequency selectivity in the parakeet studied by isointensity masking contours," J. Exp. Biol. 87, 331-342.

Saunders, J. C., Bock, G. R., and Fahrbach S. E. (1978). "Frequency selectivity in the parakeet (Melopsittacus undulatus) studied with narrow-band noise masking," Sensory Processes 2, 80-89.

Shofner, W. P., and Sachs, M. B. (1986). "Representation of a lowfrequency tone in the discharge rate of populations of auditory nerve fibers," Hear. Res. 21, 91-95.

Wilson, J. P., and Johnstone, J. R. (1975). "Basila" membrane and middle ear vibration in guinea pig measured by sapacitive probe," J. Acoust. Soc. Am. 57, 705-723.

Winter, I. M., and Palmer, A. R. (1991). "Intensity coding in lowfrequency auditory nerve fibers of the guinea pig," J. Acoust. Soc. Am. 90, 1958-1967.

Zwicker, E. (1970). "Masking and psychological excitation as consequences of the ear's frequency analysis," in Frequency Analysis and Periodicity Detection in Hearing, edited by R. Plomp and G. F. Smoorenburg (Sijthof, Leiden), pp. 376-396 